\title{
Comparative accuracy of a shoulder range motion measurement sensor and Vicon 3D motion capture for shoulder abduction in frozen shoulder
}

\author{
Chanhee Park ${ }^{\mathrm{a}, \mathrm{b}}$, Yeongsang An ${ }^{\mathrm{b}}$, Hyunsik Yoon ${ }^{\mathrm{c}}$, Ilbong Park ${ }^{\mathrm{d}}$, Kyoungtae Kim ${ }^{\mathrm{e}}$, \\ Chungyoo $\mathrm{Kim}^{\mathrm{f}}$ and Youngjoo Cha ${ }^{\mathrm{d}, *}$ \\ ${ }^{a}$ Department of Physical Therapy, Yonsei University, Wonju, Korea \\ ${ }^{\mathrm{b}}$ Funrehab Co., Ltd, Daejoeon, Korea \\ ${ }^{\mathrm{c}}$ Department of Physical Therapy, Chungnam National University Hospital, Daejeon, Korea \\ ${ }^{\mathrm{d}}$ Department of Sports Rehabilitation, Busan University of Foreign Studies, Busan, Korea

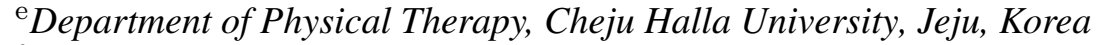 \\ ${ }^{\mathrm{f}}$ Department of Rehabilitation Medicine, Busan St. Mary's Hospital, Busan, Korea
}

\begin{abstract}
.
BACKGROUND: Although patients with frozen shoulders have the range of motion (ROM) of their shoulder's abduction movements measured at hospital and the physical therapy visits, multiple visits to check for progress is often difficult. Thus, we developed an artificial intelligence-based image recognition detectable sensor (AIRDS) intended for easy use at home.

OBJECTIVE: The purpose of this study was to determine the accuracy of a sensor (AIRDS) measuring shoulder abduction angle, thus offering a valid and feasible system for monitoring patients with frozen shoulder.

METHODS: Ten patients with frozen shoulder ( 5 males, 5 females) performed shoulder joint movements while being measured with the AIRDS system and the 3-dimensional Vicon system. The measure of the outcome included the linear regression of the shoulder abduction joint kinematics.

RESULTS: Linear regression analysis of the AIRDS system and the Vicon system demonstrated a significant correlation coefficient of $R^{2}=0.9979(P<0.05)$.

CONCLUSIONS: Our results provide novel, promising evidence that AIRDS can accurately measure the timing and total spatial characteristics of clinical movements. AIRDS is designed to provide real-time ROM measurements for joint mobility using artificial intelligence instead of the judgement of the physical therapist.
\end{abstract}

Keywords: Shoulder abduction, convolutional neural network, artificial intelligence, frozen shoulder

\section{Introduction}

Patients with frozen shoulder have a limited range of motion (ROM) in shoulder abduction. Adhesive capsulitis is a symptom of frozen shoulder and can cause stiffness and pain in the shoulder joint, decreasing

\footnotetext{
${ }^{*}$ Corresponding author: Youngjoo Cha, Department of Physical Therapy, Cheju Halla University, 38, Halladaehak-ro, Jeju-si, Jeju-do, Korea. E-mail: chazoo0849@ chu.ac.kr.
} 
the ability to move the arm in a range of multidirectional motions [1,2]. Physical therapy is necessary for patients who demonstrate limited ROM or movement because of conditions such as frozen shoulder and adhesive capsulitis [3-6]. However, there are limitations to physical therapy, and it is difficult to measure the ROM of the shoulder every day. A self-managed shoulder abduction ROM assessment program for people with frozen shoulder is feasible using artificial intelligence-based ubiquitous healthcare services. Rapid innovations in technology have facilitated the study of human movements, and the technology of detecting body movements is widely applicable in healthcare, allowing patients to be monitored and rehabilitated from home. Motion analysis involves tracking parts of a moving body and calculating data based on sequential images of bodily movements [7-9].

Convolutional Neural Network (CNN) algorithm approaches were implemented to classify human movements, including movements of the head, arms, torso, or legs. The CNN algorithms enable the analysis of images of bodily gestures and can be used to support assisting interfaces, such as healthcare monitoring systems [10]. So, we developed an artificial intelligence (AI)-based image recognition detectable sensor (AIRDS) system using CNN algorithm to measure abduction in frozen shoulder patients. The AIRDS system uses novel intelligence image recognition and is designed to provide real-time ROM measurement of joint mobility through AI instead of the assessment of the physical therapist; patients can use AIRDS with a webcam and desktop, a smartphone, or a laptop with a camera. The purpose of this study was to develop and validate a practical tool that allows clinicians to measure shoulder abduction in clinical setting using smartphone. Our vision is that this system will provide reliable, automatic analysis of angle kinematics that is sensitive, accurate, robust, and consistent, without visiting the hospital. To achieve this objective, we carried out a study in order to determine: the validity of AIRDS compared to Vicon 3-D motion analysis of angle kinematics. We hypothesized a subset of most sensitive, reliable and valid AIRDS measure to characterize joint kinematics in participants with limited shoulder abduction.

\section{Methods}

\subsection{Participants}

Ten patients with frozen shoulder (mean age $=58.3 \pm 4.7$ ) were volunteered in this study. The study was approved by the Research Ethics Committee (20200715-HR-55-04-06) Institutional Review Board of the Republic of Korea. Written informed consent was obtained from participants. The inclusion criteria included (1) age 40 to 70 years, (2) local shoulder pain, (3) pain was frequently present either over the anteromedial aspect of the shoulder extending distally into the biceps region or over the lateral aspect of the shoulder extending into the lateral deltoid region, (4) symptoms had been present for at least three months, (5) loss of active and passive global shoulder motion, with at least $50 \%$ loss of external rotation. The exclusion criteria were as follows: (1) pathologic findings or glenohumeral osteoarthritis on radiographic evaluation, (2) clinical evidence of cervical spine disease, (3) trauma to the shoulder, (4) local corticosteroid injection or any physiotherapy intervention to the affected shoulder within the last three months, (5) cerebrovascular accident affecting the shoulder, (6) inflammatory joint disease affecting the shoulder, (7) bilateral frozen shoulder due to a possible underlying systemic cause, or (8) prior surgery, dislocation, or fractures on the affected shoulder [11].

\subsection{Study design}

The study was performed in the motion analysis laboratory, to determine experimental concurrent 
Table 1

\begin{tabular}{cl}
\multicolumn{2}{c}{ Attachment of the sensors to the body } \\
\hline Id & \multicolumn{1}{c}{ Part } \\
\hline 0 & Nose \\
1 & Left eye \\
2 & Right eye \\
3 & Left ear \\
4 & Right ear \\
5 & Left shoulder \\
6 & Right shoulder \\
7 & Left elbow \\
8 & Right elbow \\
9 & Left wrist \\
10 & Right wrist \\
11 & Left hip \\
12 & Right hip \\
13 & Left knee \\
14 & Right knee \\
15 & Left ankle \\
16 & Right ankle \\
\hline
\end{tabular}

validity of AIRDS compared to 3-dimensional Vicon motion capture system analysis (Vicon MX, Oxford, UK). The Vicon 3D motion analysis demonstrated superior reliability [intraclass correlation coefficients $(\text { ICC })_{3, k} r=0.998$ ] and accuracy (standard error of the mean $=1.83$ ) in the measurement of complex movements, making it suitable to use for our criterion measures [12]. The AIRDS systems permit the calculation of 2D position and angles. Participants' abduction of the affected shoulder until painful in 3 repetitions. Participants took a 10-minute break during each experiment.

\subsection{Instruments}

The AIRDS system takes advantage of the fact that a CNN is an AI learning network that allows a computer to mimic human neural networks. Previous research has considered using AI algorithms with MobileNet and ResNet to learn poses. Using MobileNet would make the process lighter, but future research could use ResNet to grow our project and improve accuracy. Studies show MobileNet to be small, fast, and inaccurate, but ResNet has been found to be big and slow but accurate [13]. AI can be divided into supervised learning and unsupervised learning. This research involved learning the system using 10,000 pictures of each angle as supervised learning [14,15]. To increase measured shoulder abduction the recognition rate, the patient's eyes, nose, mouth, and ankle were also used in the calculations. Additional advantages of our system include affordability, portability, and accessibility. The most important functional features of the AIRDS system compared to the specializing in changing the abduction angle of patients with frozen shoulder. Table 1 lists the identification number of the sensor and where it was attached to the body. The AIRDS sensors were attached to the face to determine the direction of the face, and a sensor was also recognized on the hips, knees, and ankles.

\subsection{Experimental procedures}

Participants performed shoulder joint functional movements while being concurrently measured by the AIRDS system and the Vicon motion capture system. Participants stood with the sensors of our system attached directly facing the smartphone camera at a distance of $1 \mathrm{~m}$, which is adequate to collect accurate data. The smartphone camera was located $2 \mathrm{~m}$ from the ground, with the lens vertical to the floor and 


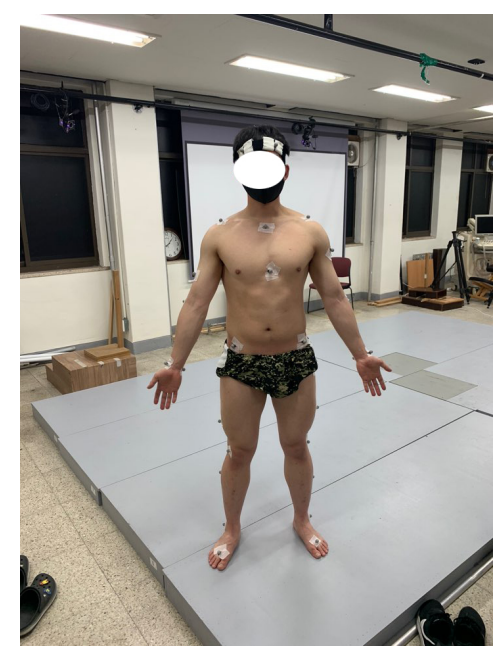

Fig. 1. Plug-in-gait full body marker set from Vicon.

pointing towards the participant. The investigator $(\mathrm{CH})$ stood beside the smartphone with AIRDS to show the movements. The participant's movements included standing still and the abduction of the affected shoulder until painful.

\subsection{Data processing}

Vicon data was filtered using the finite impulse response (FIR) filter and processed using the plug-in-gait full body marker set in Vicon Nexus (Fig. 1). Two extra markers were positioned on the fingernail of the thumb and index finger to measure hand motion. We also measured kinematic measurements using Vicon. The Vicon motion capture system sampled at $120 \mathrm{~Hz}$ using Vicon Nexus 1.7 software. The AIRDS system uses representative kinematic data established by Neumann's kinesiology [16]. Maximum angular movement for shoulder abduction was determined using a custom script within LabVIEW software (LabVIEW version 7.5, National Instruments Corporation, Austin, TX, USA).

\subsection{Statistical analysis}

Linear regression analysis and coefficient of variation statistics were computed to determine the validity and variation of the AIRDS system. A power analysis, using G-Power software, was conducted to assess the sample size requirement $(N=8)$, based on our pilot study which demonstrated effect size (Eta squared, $\left.\eta^{2}=1.02\right)$ and power $(1-\beta=0.8)$. Significance was assigned at a $\mathrm{P}$ of less than 0.05 for all analyses. Statistical analyses were performed with SPSS/Windows (version 25.0).

\section{Results}

All participants who completed the process of assessment were included in the analysis. Table 2 summarizes the demographic and clinical characteristics of the participating patients.

\subsection{Angular kinematic data}

To determine the validity of the AIRDS system, we compared joint angle data obtained from both 
Table 2

Demographic characteristics of the subjects $(N=10)$

\begin{tabular}{ll}
\hline \multicolumn{1}{c}{ Variables } \\
\hline Age (years) & $58.3 \pm 4.7$ \\
Onset (months) & $7.8 \pm 2.7$ \\
Affected side (Lt/Rt) & $7 / 3$ \\
Male (\%) & 50 \\
Female (\%) & 50 \\
\hline
\end{tabular}

Lt, left; Rt, Right.

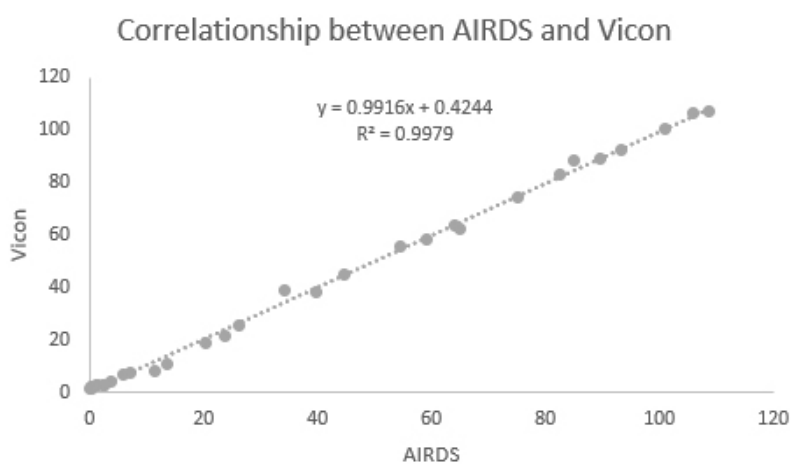

Fig. 2. Linear regression of independent shoulder abduction angle data obtained from AIRDS and Vicon measurements. AIRDS, artificial intelligence-based image recognition detectable sensor.

AIRDS and Vicon measurements. Figure 2 illustrates the linear regression between the two measured angles, demonstrating an excellent correlation for shoulder abduction $\left(R^{2}=0.9979, P<0.05\right)$ angle data, thus supporting the validity and accuracy of the AIRDS system.

\section{Discussion}

The present study investigated the comparative accuracy of the AIRDS system and Vicon 3D in measuring shoulder abduction in patients with frozen shoulder. As hypothesized, the measurements of all participants showed superior correlation results of angle joint kinematics. This novel approach provides hopeful evidence of the significant therapeutic benefits of this system for measuring ROM in frozen shoulder patients. AIRDS is an innovative tool that can allow clinicians to objectively measure shoulder abduction during stance easily and quickly in all setting. The approach of using CNN algorithms to quantify shoulder abduction was shown to be sensitive, valid, and reliable for participants with limited shoulder abduction.

The previous study by Galna et al. showed good agreement between the Kinect and Vicon systems in the measurement of shoulder abduction in people with Parkinson's disease [17], and previous other studies have been able to broaden our knowledge on the accuracy of the Kinect system for measuring upper and lower body kinematics $[18,19]$. These studies show that Kinect was able to measure small movements reasonably well, such as hand clasping, but was not as successful with larger spatial characteristics; the system found it difficult to measure fine motor movements. Kinect was unable to collect spatial characteristics with the same accuracy as timing characteristics. For example, a study found that the ICC for all temporal attributes were higher than 0.9, but the range of operations was between 0.009 and 0.989 [20]. 
The present study showed that the relationships between measurements (linear regression) were largely strong. This suggests that the AIRDS system may be most useful for measuring the slowness of movement in people with frozen shoulder, rather than measuring the decrease in the size of movement.

Furthermore, although the measurement of the range of motion was not as accurate as that of Vicon, the AIRDS system may still be useful for tracking changes in people's motion over time, such as motor dysfunction caused by intervention. However, the reliability of the AIRDS system to measure functional movements has not been established. Further development is required to measure motion symptoms in the home environment using the AIRDS system. The accuracy of our system can be improved using better spatial resolution, more accurate estimations of anatomical landmarks, and by determining the optimal sagittal direction of the AIRDS system for people. A newer AIRDS system should improve spatial and temporal resolution to improve the accuracy of gross movement measurements, such as shoulder flexion, hip flexion, and trunk flexion, and to enable more proper anatomical models. Some of the inaccuracies of the AIRDS system may be due to the limits of the ability of the AIRDS system to estimate anatomical landmarks. Additionally, patients with frozen shoulder find it difficult to buy, install, and use the Kinect camera. The AIRDS system makes the process easier by allowing access to it through a smartphone camera.

\section{Conclusion}

The AIRDS system has the potential to be a safe, accurate, low-cost, and home-based sensor for measuring the movements of people with frozen shoulder. The AIRDS system can accurately measure the timing and total spatial characteristics of clinical movements but cannot measure them with the same spatial accuracy for finem motor movements. Further development is needed to improve the tracking of smaller movements and to develop user-friendly software to monitor limited shoulder range at home. Robust experimental research with larger sample sizes in both normal and age-matched orthopedic populations is now warranted to prove the reliability of our system.

\section{Acknowledgments}

This work was supported by a research grant of the Busan University of Foreign Studies in 2022.

\section{Conflict of interest}

None to report.

\section{References}

[1] Sharma RK, Bajekal RA, Bhan S. Frozen shoulder syndrome. International Orthopaedics. 1993; 17(5): $275-278$.

[2] Bunker TD, Anthony PP. The pathology of frozen shoulder. A dupuytren-like disease. The journal of bone and joint surgery. British volume. 1995; 77(5): 677-683.

[3] Neviaser RJ, Neviaser TJ. The frozen shoulder. Diagnosis and management. Clinical Orthopaedics and Related Research. 1987; (223): 59-64.

[4] Ma SY, Je HD, Jeong JH, et al. Effects of whole-body cryotherapy in the management of adhesive capsulitis of the shoulder. Archives of Physical Medicine and Rehabilitation. 2013; 94(1): 9-16. 
[5] Shin SJ, Lee SY. Efficacies of corticosteroid injection at different sites of the shoulder for the treatment of adhesive capsulitis. Journal of Shoulder and Elbow Surgery. 2013; 22(4): 521-527.

[6] Arslan S, Çeliker R. Comparison of the efficacy of local corticosteroid injection and physical therapy for the treatment of adhesive capsulitis. Rheumatology International. 2001; 21(1): 20-23.

[7] Lin HC, Chiang SY, Lee K, et al. An activity recognition model using inertial sensor nodes in a wireless sensor network for frozen shoulder rehabilitation exercises. Sensors. 2015; 15(1): 2181-2204.

[8] Crane E, Gross M. Motion capture and emotion: Affect detection in whole body movement. In International Conference on Affective Computing and Intelligent Interaction (pp. 95-101). Springer, Berlin, Heidelberg, 2007.

[9] Jolles BM, Duc C, Coley B, e al. Objective evaluation of shoulder function using body-fixed sensors: A new way to detect early treatment failures. Journal of Shoulder and Elbow Surgery. 2011; 20(7): 1074-1081.

[10] Ijjina EP, Chalavadi KM. Human action recognition using genetic algorithms and convolutional neural networks. Pattern Recognition. 2016; 59: 199-212.

[11] Russell S, Jariwala A, Conlon R, et al. A blinded, randomized, controlled trial assessing conservative management strategies for frozen shoulder. Journal of Shoulder and Elbow Surgery. 2014; 23(4): 500-507.

[12] Pfister A, West AM, Bronner S, et al. Comparative abilities of Microsoft Kinect and Vicon 3D motion capture for gait analysis. Journal of Medical Engineering \& Technology. 2014; 38(5): 274-280.

[13] Kim W, Dehghan F, Cho S. Vehicle License Plate Recognition System using SSD-Mobilenet and ResNet for Mobile Device. Smart Media Journal. 2020; 9(2): 92-98.

[14] Zhang Y, Xiang, T, Hospedales TM, Lu H. Deep mutual learning. In Proceedings of the IEEE Conference on Computer Vision and Pattern Recognition (pp. 4320-4328), 2018.

[15] Weng TW, Zhang H, Chen PY, et al. Evaluating the robustness of neural networks: An extreme value theory approach. arXiv preprint arXiv1801.10578, 2018.

[16] Neumann DA. Kinesiology of the musculoskeletal system-e-book: foundations for rehabilitation. Elsevier Health Sciences, 2013.

[17] Galna B, Barry G, Jackson D, et al. Accuracy of the Microsoft Kinect sensor for measuring movement in people with Parkinson's disease. Gait \& Posture. 2014; 39(4): 1062-1068.

[18] Clark RA, Pua YH, Fortin K, et al. Validity of the Microsoft Kinect for assessment of postural control. Gait \& Posture. 2012; 36(3): 372-377.

[19] Fern'ndez BA, Susín A, Lligadas X. Biomechanical validation of upper-body and lower-body joint movements of kinect motion capture data for rehabilitation treatments. In 2012 fourth international conference on intelligent networking and collaborative systems (pp. 656-661). IEEE, 2012.

[20] Obdržálek Š, Kurillo G, Ofli F, et al. Accuracy and robustness of Kinect pose estimation in the context of coaching of elderly population. In 2012 Annual International Conference of the IEEE Engineering in Medicine and Biology Society (pp. 1188-1193). IEEE, 2012. 